\title{
ABJS Carl T. Brighton Workshop on Health Informatics in Orthopaedic Surgery
}

\section{Editorial Comment}

\author{
Christian J. H. Veillette MD, MSc, FRCSC
}

Published online: 7 July 2010

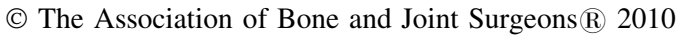

The economic woes of the United States (U.S.) healthcare system have given rise to an unprecedented federal effort to modernize the information systems and promote the adoption of health information technology (HIT). The recent economic stimulus package- the American Recovery and Reinvestment Act of 2009 (ARRA) - has a HIT component (HITECH Act) that will allocate $\$ 17$ billion in financial incentives intended to persuade doctors and hospitals to adopt meaningful use of an electronic health record (EHR) and \$2 billion for support systems and requisite infrastructure [1,2]. The purpose of the HITECH Act is to encourage health care providers to leverage HIT tools to achieve quality and efficiency goals. Although this primary value proposition for HIT adoption by health care professionals remains a matter of debate, the potential for secondary benefits such as the reuse of clinical data for research and quality improvement is inevitable [8].

The U.S. is not alone in their efforts to adopt HIT. The United Kingdom's NHS Connecting for Health (http://www.informatics.nhs.uh), Australia's HealthConnect (www.healthconnect.gov.au) and Canada's Health Infoway (www.infoway-inforoute.ca) represent three such efforts at different stages of implementation. EHRs, however, are just one of the many tools of health informatics. The tools of informatics encompass clinical guidelines and decision support, formal health languages, information systems (EHRs, PACS, integrated databases and registries) and communication systems (Internet, telemedicine). It is

C. J. H. Veillette $(\bowtie)$

Division of Orthopaedic Surgery, University of Toronto, Toronto

Western Hospital, 399 Bathurst Street, Toronto, ON M5T 2S8, Canada

e-mail: christian.veillette@uhn.on.ca; orthonet@gmail.com important to remember that these tools are only a means to an end - the delivery of the best possible healthcare.

Informatics is the intersection of people, information and technology. Within the field of informatics are several major subcategories, each with their own domain (Fig. 1). Health informatics has been defined broadly as the logic of healthcare [3]. It is a field concerned with the optimal use of information, often aided by technology, to improve individual health, health care, public health, and biomedical research [6].

Substantial differences have been identified between various clinical fields to justify the creation of areas of "subspecialty" informatics. Certainly there is enough uniqueness in our information needs, those of our patients and the technologies we routinely use to make clinical decisions to warrant the existence of orthopaedic informatics. Orthopaedic informatics is therefore the logic of orthopaedics - the rational study of the way we think about patients: the way we define, select and evolve treatments; how we create, share and apply clinical knowledge; the information needs of our consumers. It is aided by informatics tools to obtain the information we need in the appropriate detail, of the appropriate quality, when we need it, where we need it, to improve the efficiency and effectiveness of patient care, research and education. Informatics skills underpin communicating effectively, structuring information, questioning to find information, searching for knowledge and making decisions. Informatics has been recognized as a core competency for patientcentered care and the requirements of a changing health system [5]. It is important to note that informatics competency is not just computer literacy.

The 2009 ABJS Carl T. Brighton Workshop on Health Informatics in Orthopaedic Surgery brought together various stakeholders in health informatics from across the 


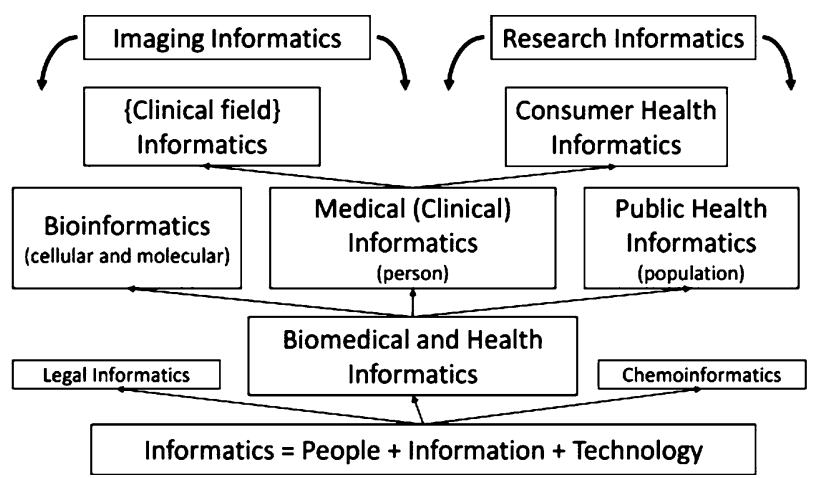

Fig. 1 Major subcategories of informatics. (Reprinted with courtesy from Hersh W. A stimulus to define informatics and health information technology. BMC Med Inform Decis Mak. 2009;9:24.)

World to present, debate and develop recommendations for advancing the field of orthopaedic informatics. Representatives from health care institutions, surgeons, HIT vendors, researchers, informaticians, regulators and policymakers, and payers participated in the active discussions on the eight major themes covered including (1) patient education and the Internet; (2) Internet-based education and simulation; (3) telemedicine, disparities in utilization, access to information communication technology (ICT); (4) Web 2.0 and publishing; (5) ontologies and search; (6) informatics in orthopaedic training; (7) information systems, databases, registries - aligning data models in orthopaedics; and (8) EHRs in orthopaedics. We asked each of the Workshop participants to provide constructive feedback with the objective to create summary recommendations and answer the three questions originally asked by Dr. Carl Brighton who established these Workshops in 1996: (1) Where are we now? (2) Where do we need to go? and (3) How do we get there?

\section{Patient Education and the Internet}

The Internet has become the initial point of contact for patients who are researching health issues and treatment options. Social networking and the ability of patients to effortlessly find individuals with similar problems has led to the creation of online communities with a wealth of outcome information. The ability to tap into these communities and provide a standardized user interface for data collection remains to be explored in orthopaedics. Although health information literacy is the single best predictor of an individual's health status, little is known about the information needs and literacy of patients with musculoskeletal problems. Most of the patient education materials on orthopaedic websites are too advanced and require readability assessments. An improved understanding of the health literacy of specific patient populations is

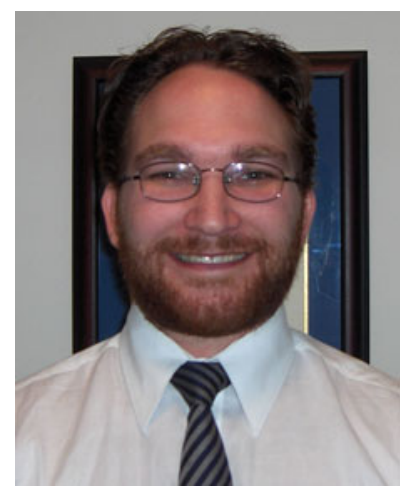

Fig. 2 Christian Veillette, MD is shown.

required, guidelines need to be created to match these levels and an opportunity exists for the creation of personalized health literacy agents that interact with the Internet on behalf of patients.

\section{Internet-based Education and Simulation}

The Internet is quickly becoming a preferred method of CME with online textbooks, webcasts and rich multimedia becoming more prominent as broadband connections become commonplace. This new pedagogy decreases barriers to information and allows more effective use of just-in-time learning principles rather than traditional justin-case methods. The most effective ways to introduce these new technologies into orthopaedic curricula requires further evaluation. In addition, multimedia simulation and virtual reality simulators have demonstrated the potential to become an integral part of teaching and quality improvement initiatives. However, the specific circumstances where one method is favored over the other requires the development of standardized outcome tools to allow direct comparisons between the two technologies and evaluation of the cost-benefit ratio for training.

\section{Telemedicine, Disparities in Utilization, Access to ICT}

There are large disparities in the utilization of HIT. Internet and mobile phone access to journals and clinical resources potentially revolutionizes access to information for patient care, education and research in low income countries. An improved understanding of the orthopaedic information needs and access barriers in developing worlds is much needed. The promotion of open access publications or alternative models to provide resources for free or at low cost to doctors in low income countries may allow increased knowledge transfer and improved quality of care. 
In addition, administrative databases can be coupled with incentives to hospitals and/or surgeons to supply implants only if the cases are reported. However, the barriers to routine clinical followup need to be removed and low cost but widespread data capture/transfer mechanisms using mobile phone technology are required.

\section{Web 2.0 and Publishing}

The Internet has evolved from an information-retrieval, read-only form to a more interactive, dynamic and participatory form called Web 2.0, which allows users to generate and share content. This read/write web is built upon new technologies and approaches. Use of these Web 2.0 tools to promote collaboration between patients, their caregivers, medical professionals, and other stakeholders in health is collectively called Health 2.0. Orthopaedic surgeons must embrace the rise of participatory medicine [9], become active in the use of these technologies and guide their development or risk being overwhelmed by wellmeaning, but scientifically flawed, consumer-generated information.

Despite the extraordinary success of Wikipedia and the potential of wiki publishing, its adoption in orthopaedics has been limited. In contrast to conventional static publications, dynamic publications in the wiki model have no final version, no definite date of publication and continuous integration and synthesis of new findings and insights. A collaborative orthopaedic knowledgebase developed using wiki technology can provide a rigorous educational and research publishing platform if the barriers to collaborative authorship, credibility, and acceptance of open communitydriven publication models can be addressed.

\section{Ontologies and Search}

The Semantic Web describes methods and technologies to allow machines to understand the meaning ("semantics") of information on the Internet. These technologies require machine-readable metadata that enables automated agents and other software to access the Web more intelligently. The agents would be able to perform tasks automatically and locate related information on behalf of the user. Ontologies are common vocabularies that not only define a concept but also the relationships between concepts. For orthopaedics to benefit from the potential of semantic technologies such as standardized data collection, linked data sources, knowledge translation, information retrieval, and trust management, orthopaedic specific ontologies need to be defined and evaluated.

\section{Health Informatics and IT in Orthopaedic Training}

Electronic logbooks of surgical experience for orthopaedic trainees allows for objective assessment of the number or type of procedures that the average trainee performs for each operation and the creation of normative datasets across orthopaedic training programs. The comparison across regions can improve the overall quality of training and highlight gaps in experience. The logbook can be combined with longitudinal procedure based assessment tools to create a profile of the trainee's level of competence. In addition, IT tools can be used to improve the quality and efficiency of patient handoff and mitigate the potential increased risk of error due to increases in the number of patient handoffs caused by resident duty hour restrictions.

The key principles of evidence-based medicine (EBM) are driven by informatics skills; health informatics can be considered the engine driving EBM. The time and resources spent teaching the EBM methodology may be better spent on information management (IM). IM strives to deliver just-in-time information that can be effectively and efficiently used in face-time with patients.

\section{Information Systems, Databases, Registries-Aligning Data Models in Orthopaedics}

Current HIT implementation in orthopaedics often creates siloed, disparate, and unstructured data that cannot be readily translated into useful knowledge. The standardization of data collection procedures and definition of common data elements across current outcome tools is required for the effective, valid and reliable collection of outcome data for the orthopaedic population. Although commercial electronic data capture (EDC) software is widely available, it is often cost- and resource-intensive, lacks customizability and is difficult to integrate into existing IT platforms due to its proprietary nature. Open source web-based EDC software can overcome these limitations and provide a foundation and development platform for a standardized orthopaedic outcomes engine. The automated integration of data from disparate source such as operative notes, anesthesia information systems, electronic clinical documentation, and PACS minimizes input from physicians, increases compliance and improves large scale data collection efforts.

\section{EHRs in Orthopaedics}

Computing technology has transformed virtually every aspect of our daily life, however the proportion of U.S. 
health care professionals and hospitals that have transitioned to EHRs is remarkably small [4, 7]. The barriers include cost, technical challenges, interoperability, privacy and confidentiality and an adequate HIT workforce. A formal needs assessment and survey of the orthopaedic community is required to understand our specific EHR needs and experiences with the HIT landscape. Guidelines on the reuse of clinical data for research and quality improvement must be established alongside the mapping and standardization of datasets to allow distributed orthopaedic data networks to be developed. The use of Web 2.0 strategies with user generated reviews and ratings of EHR and HIT vendors may provide an ongoing understanding of the orthopaedic EHR environment.

Several recurrent themes surfaced over the course of the Workshop. Informatics involves the complex interaction between people, information and technology. The technological challenges involved often are undermined by the many socio-organizational or "people" issues that must be understood and addressed to enable and adopt HIT advances. A major collaborative effort by many of the stakeholders involved, including health care institutions and surgeons, HIT vendors, researchers, informaticians, regulators and policymakers, payers, and patients, is required to realize the full promise of health informatics in orthopaedic surgery. Orthopaedic surgeons with informatics skills are required to navigate the changing healthcare environment and translate HIT investments into improved patient care, education and research.

In true informatics fashion, a collaborative wiki workspace was created for the Workshop to allow the participants to create a repository and starting point for ongoing interaction. The workspace is available on Orthopaedia $\mathbb{R}$ at http://www.orthopaedia.com/display/HIOS/Home and contains participant profiles, abstracts, presentations, discussions, and additional recommendations. To view the workspace signup for a free membership at http://www. orthopaedia.com.
I would like to acknowledge Dr. Vincent Pellegrini and the ABJS Board for their insight in selecting a timely symposium topic that is early in its development and often not discussed at orthopaedic meetings. I would also like to thank the CORR Editorial Staff and the reviewers who made constructive comments and improvements to the manuscripts contained in this symposium. Finally, I would like to thank the participants of the 2009 ABJS Carl T. Brighton Workshop on Health Informatics in Orthopaedic Surgery for their time, commitment, openness, and constructiveness. Together we have taken great strides to define the scope and provide direction for the field of Orthopaedic Informatics.

\section{References}

1. Blumenthal D. Launching HITECH. N Engl J Med. 2009;362:382385 .

2. Blumenthal D. Stimulating the adoption of health information technology. N Engl J Med. 2009;360:1477-1479.

3. Coiera E. Guide to Health Informatics. 2nd ed. Oxford, UK: A Hodder Arnold Publication; Oxford University Press; 2003.

4. DesRoches CM, Campbell EG, Rao SR, Donelan K, Ferris TG, Jha A, Kaushal R, Levy DE, Rosenbaum S, Shields AE, Blumenthal D. Electronic health records in ambulatory care-a national survey of physicains. $N$ Engl J Med. 2008;359:50-60.

5. Greiner AC, Knebel E (eds); COTHPE, BOHC Services. Health Professions Education: A Bridge to Quality. Washington, DC: Institute of Medicine National Academies Press; 2003.

6. Hersh W. A stimulus to define informatics and health information technology. BMC Med Inform Decis Mak. 2009;9:24.

7. Jha AK, DesRoches CM, Campbell EG, Donelan K, Rao SR, Ferris TG, Shields A, Rosenbaum S, Blumenthal D. Use of electronic health records in U.S. hospitals. $N$ Engl J Med. 2009;360:1628-1638.

8. Weiner MG, Embi PJ. Toward reuse of clinical data for research and quality improvement: the end of the beginning? Ann Intern Med. 2009;151:359-360.

9. Why Healthcare Professionals Should Practice Participatory Medicine: Perspective of a Long-Time Medical Editor. 2009. Journal of Participatory Medicine (JoPM) Web site. Available at: http://jopm.org/index.php/jpm/article/viewArticle/31/23. Accessed June 16, 2010. 